\title{
Ge(113) reconstruction stabilized by subsurface interstitials: An x-ray diffraction structure analysis
}

\author{
H. Vogler, A. Iglesias, and W. Moritz \\ Institut für Kristallographie der Universität München, Theresienstrasse 41, D-80333 München, Germany \\ H. Over \\ Fritz-Haber-Institut der Max-Planck-Gesellschaft, Faradayweg 4-6, D-14195 Berlin, Germany
}

(Received 22 September 1997)

\begin{abstract}
The three-dimensional atomic coordinates of the Ge(113)- $(3 \times 1)$ surface have been determined by analyzing in-plane and out-of-plane x-ray intensity data. Besides dimer and adatom motifs, which reduce the number of dangling bonds, a random distribution of subsurface interstitials has been identified. Subsurface interstitials relieve elastic stress and lower the energy of the electronic system. Together with the delicate balance between the energy gain due to reduction of dangling bonds and the energy costs due to induced strain this determines the nature of the $(3 \times 1)$ reconstruction of $\mathrm{Ge}(113)$. [S0163-1829(98)02003-7]
\end{abstract}

The (113) surfaces of Si and Ge are distinctively stable $\mathrm{e}^{1,2}$ revealing a surface energy almost as low as that found for the [001] orientation. $^{3}$ To attain this low surface energy, both (113) surfaces undergo heavy reconstructions exhibiting either $(3 \times 1)$ or $(3 \times 2)$ periodicity depending on the temperature. ${ }^{4}$ Until now the atomic geometry of these surfaces and the structural elements stabilizing this orientation have not been identified unambiguously. In principle it can be expected that the same structural elements as found on the (001) and the (111) faces are also operative at the (113) orientation, since the bulk-truncated (113) surface consists of atoms with one and with two dangling bonds.

The stabilization of the low-index (001) and (111) surfaces of elemental semiconductors is well understood, and is mainly controlled by the reduction of the number of dangling bonds at the surface without introducing too much strain. ${ }^{5}$ Mainly two structural elements meeting this principle have been identified so far. Most notably, the so-called adatoms are able to saturate three dangling bonds on (111) surfaces while creating only one dangling bond. This structural motif is known to stabilize the clean $\mathrm{Ge}(111)-c(2 \times 8)$ surface, ${ }^{6}$ and is also a major stabilizing factor of the dimer-adatomstacking-fault (DAS) model of the clean $\mathrm{Si}(111)-(7 \times 7)$ surface. ${ }^{7}$ The second structural element that effectively reduces the number of dangling bonds is found on (001) surfaces, whose bulk-truncated structure consists of atoms carrying two unsaturated bonds. In pairing up two adjacent atoms, i.e., forming so-called dimers, the number of dangling bonds can be halved; this structural element was first proposed by Schlier and Farnsworth. ${ }^{8}$

The bulk-truncated (113) surfaces of Si and Ge expose two types of atoms: one with two dangling bonds as on the (001) surface, and one with one dangling bond as on (111). Applying the above-mentioned simple principle for the stabilization of semiconductor surfaces, Ranke ${ }^{9}$ proposed several reasonable structure models among which one was recently shown by a low-energy electron-diffraction (LEED) structure analysis to describe the atomic geometry of the $\mathrm{Si}(113)-(3 \times 1)-\mathrm{H}$ surface. ${ }^{10}$ The main structural elements of this model consist of dimers formed by two out of three (001)-like atoms and of (111)-like atoms which act as adatoms if the attached unpaired (001)-like atom is removed. The resulting structure then has $(3 \times 1)$ periodicity containing one dimer and two adatoms per $(3 \times 1)$ unit cell (referred to as the DA model). The dimer is part of a (more or less) coplanar five-atom ring which is called a pentamer in the following. When the clean $(3 \times 2)$ surface is exposed to atomic hydrogen at room temperature, the $(3 \times 1)-\mathrm{H}$ structure is formed without substantial mass transport. ${ }^{11}$ Therefore, it can be concluded that the structural building blocks of the $(3 \times 1)-\mathrm{H}$ are also to be present in the clean $(3 \times 2)$ reconstruction. However, this structure model is far from being compelling, since the number of dangling bonds is still quite large, and the adatoms induce marked tensile stress into the surface region. This situation may call for an additional structural unit which is mobile enough to account for the ready transition between these $(3 \times 2)$ and $(3 \times 1)$ reconstructions, and which further reduces the surface energy. On the basis of a combined investigation using density-functionaltheory (DFT) calculations and scanning tunneling microscopy $(\mathrm{STM})^{3}$ this missing structural element has been proposed to be interstitial atoms beneath every second pentamer although very recently this model has been questioned by several authors. ${ }^{12,13}$

Up to now, this structural element has not been identified with a surface-crystallographic method. The present surface$\mathrm{x}$-ray-diffraction (SXRD) structure analysis will fill this gap by supplying direct evidence of these interstitial atoms and, in addition, providing detailed three-dimensional crystallographic data of the clean Ge(113) surface. We chose Ge instead of $\mathrm{Si}$ since this substrate is easier to measure with SXRD due to its stronger scattering power. Generally, structural results of Ge surfaces agree quite well with corresponding results of Si counterparts, taking the different lattice constants into account. Therefore, the structural results presented here for the $\mathrm{Ge}(113)$ surface should be directly transferable to the case of the $\mathrm{Si}(113)$ surface.

A commercially available Ge waver (not doped) was cut along the [113] direction, and mechanically polished using diamond paste and syton $(0.25-\mu \mathrm{m}$ granulation size). The 
a)

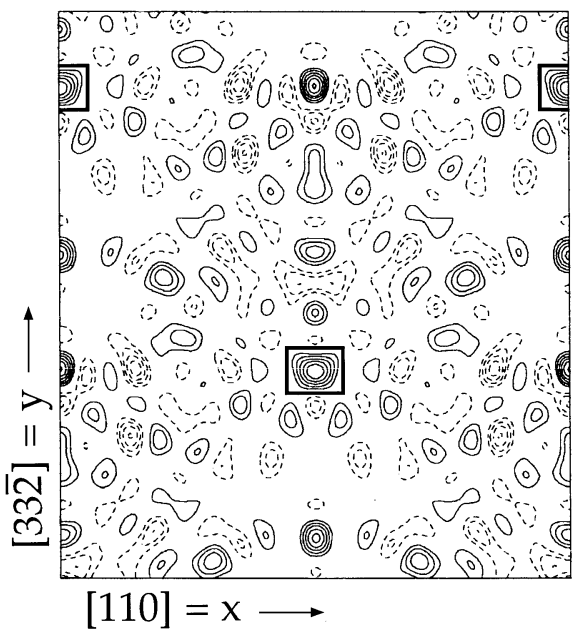

b)

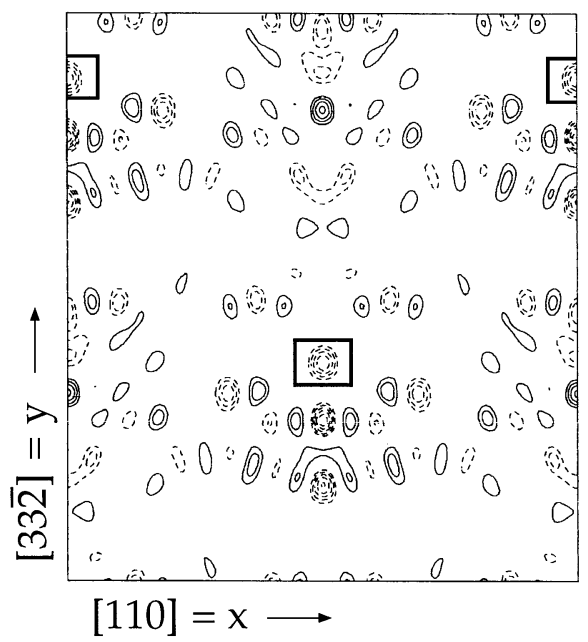

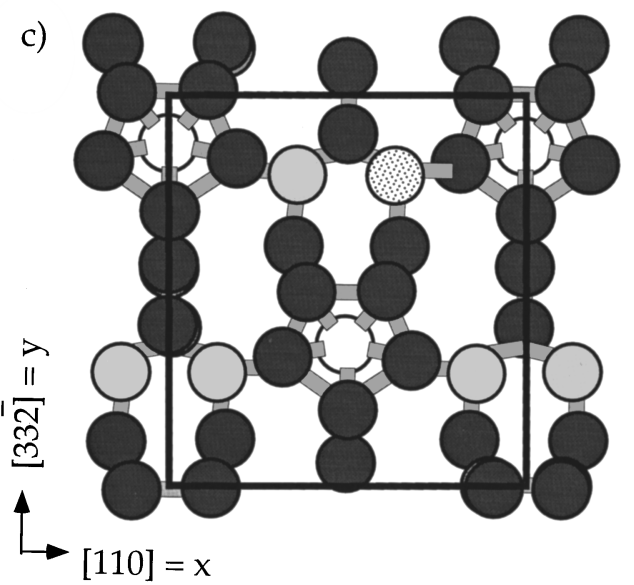

FIG. 1. (a) Fourier difference map for the Ge(113)-(3×1) DA model, i.e., no interstitial atom beneath the pentamers, and the experimental data. (b) Fourier difference map for the Ge(113)- $(3 \times 1)$ DAI model, i.e., every pentamer carries an interstial atom. Positive contours (solid lines) and negative contours (dashed line) indicate that something in the structure model is missing or too much, respectively. The distance between consecutive contour lines is $0.04 e / \AA^{3}$. The small squares indicate the position of the center of the pentamer where the interstitial atom should be located. (c) The $(3 \times 1)$ unit cell with and without interstitial atoms beneath (white spheres) the pentamers is used for the electron-density difference plots in (a) and (b).

deviation of the surface normal from the [113] direction was less than $0.1^{\circ}$. Under ultrahigh-vacuum (UHV) conditions $\left(<10^{-8} \mathrm{~Pa}\right)$ the crystal was prepared by repeated cycles of $\mathrm{Ar}^{+}$-ion bombardment at $500 \mathrm{eV}$ and $900 \mathrm{~K}$, followed by annealing at $1180 \mathrm{~K}$ for several minutes and slow cooling to room temperature. Auger measurements indicated a clean surface. At room temperature the LEED pattern of the clean $\mathrm{Ge}(113)$ surface exhibits very sharp $(3 \times 1)$ spots with very weak and diffuse $(3 \times 2)$ spots. From the width of the $(3 \times 1)$ spots in both LEED and SXRD the terrace width was estimated to be larger than $2000 \AA$.

The SXRD measurements were performed at the $W 1$ wiggler beam line of the Hamburg Synchrotron Radiation Laboratory HASYLAB with $0.32^{\circ} \mathrm{x}$-ray incidence angle using a wavelength of $1.2 \AA$. We confined the measurements to $(3 \times 1)$ spots. In total, a data set of 451 symmetrically independent $(3 \times 1)$ reflections was recorded containing 43 inplane and 408 out-of-plane reflections along 21 superstructure rods in steps of $\Delta l=0.5$. The in-plane data set was measured up to a momentum transfer of $8.6 \AA^{-1}$, corresponding to a diffraction angle of $110^{\circ}$. The diffractometer allows a maximum beam exit angle of $70^{\circ}$ which corresponds to a maximum momentum transfer perpendicular to the surface of $l=12$, i.e., $\Delta q_{z}=4 \AA^{-1}\left(a_{3}=18.76 \AA\right) .{ }^{14}$ The raw $\mathrm{x}$-ray data were corrected for the active sample area, polarization, and Lorentz factor ${ }^{15}$ prior to analysis.

We started our analysis by first considering those models reported by Ranke. ${ }^{9}$ Using the full data set, the best fit could be achieved with the DA model [(3×1)-2 in Ref. 9], which was also found for the $(3 \times 1)-\mathrm{H}$ phase on $\mathrm{Si}(113)$, arriving at a weighted $r$ factor $R_{w}$ of $18 \%$. Unfortunately, the optimized bond lengths were partly too long as, e.g., the distance between atom 5 and 17 (cf. Fig. 2). Restricting the Ge coordinates in a way that the Ge-Ge bond lengths were shorter than $2.85 \AA$ and longer than $2.25 \AA$ (bulk Ge-Ge: $2.45 \AA$ ), the best $r$ factor reached with the DA model turned out to be $28 \%$, a value which is unacceptably high. The best $r$ factors reached with the models $(3 \times 1)-1$ and $(3 \times 2)-1$ from Ref. 
10 are even worse, $34.5 \%$ and $65.5 \%$, respectively. Both values are far too high, and therefore these models can clearly be ruled out. In a next step we focused on the DA model using in-plane data only. The optimum $r$ factor between theory and experiment turned out to be $20.0 \%$. Using the calculated diffraction amplitudes we performed a difference Fourier synthesis ${ }^{15}$ from which missing structural elements may be identified. The difference Fourier plot shown in Fig. 1(a) indicates, among other maxima, a strong maximum at the position where the interstitial atoms should be located, i.e., around the center of the pentamer. The appearance of the other maxima in the difference Fourier plot indicates that not only a single structural element is missing, but that there are far-reaching relaxations within the surface, such as interstitial-atom-induced displacements. Therefore, we tested a modified DA model containing one interstitial atom under each pentamer. The best $r$ factor reached with this dimer-adatom-interstitial (DAI) model using the complete experimental data set was $22 \%$, again giving partly unrealistic bond lengths. The difference Fourier synthesis of the DAI model, however, indicates negative intensities at the positions of the interstitials, i.e., too many interstitial atoms in the structure model [cf. Fig. 1(b)]. Therefore, we considered a modified DAI model for which only a fraction of the pentamers carries a subsurface interstitial atom. Since the insertion of interstitial atoms causes the surrounding atoms to move away from their original positions, the analysis now needs the weighted mixing of diffraction amplitudes calculated separately for the configuration with and without interstitials; the weighting factor is given by the fraction of pentamers containing interstitial atoms. This is a standard procedure in three-dimensional (3D) $\mathrm{x}$-ray diffraction (the method of split positions ${ }^{16}$ ). Accordingly, the number of structural parameters to be optimized in this analysis doubles, although this does not impose a severe problem since the experimental data set is large enough (cf. the discussion below). We have to refine 32 in-plane parameters, 22 out-of plane parameters, one occupation factor, and two scaling factors (due to the different resolution of in-plane and out-of plane data). Altogether these are 59 parameters.

In the following we will elaborate on the question whether our experimental data set is sufficient to determine the atomic geometry of the DAI model with confidence. We measured the out-of-plane data along the lattice rods in steps of $\Delta l=0.5$, or $\Delta q_{z}=0.17 \AA^{-1}$. It seems plausible that the data are not all independent, but most of them are. To our knowledge, there does not exist a detailed investigation at which interval in reciprocal space the data points are independent in SXRD. In 3D structure crystallography the intensities at the Bragg points are usually considered as independent. Therefore, a very conservative estimate of the number of independent data points in SXRD might be provided by the following argument. We refined the $z$ coordinates up to about $6 \AA$. From a simple estimation (adopted from 3D x-ray crystallography) ${ }^{16}$ our experimental data set contains at least 180 independent points as the number of measured reflections due to a crystal with the lattice constants $12 \times 13.76 \times 6 \AA^{3}$ is about 180 . This number alone would allow us to determine all 59 parameters, though the redundancy is marginal if all parameters were equally important. This, however, is not the case. The number of free param- eters in the top two double layers amounts to 25 . These parameters determine essentially the structure of the DAI model, namely, the pentamer, the adatom, and the interstitial site as well as the relaxation around the interstitial atom. The structure refinement of these 25 parameters already leads to an $R$ value of 0.12 ; if all positions in the top four double layers are included, we have 59 parameters and the $r$ value drops to 0.10 . The atomic positions in the deeper layers differ only slightly from their bulk positions. The main features of the structure model are obtained from 25 parameters and at least 180 independent data points. This is a very reasonable relation even in bulk structure determination. It should also be taken into account that the additional reflections on the lattice rod between the independent ones enhance the resolution and reduce the errors. It is also interesting to note that even the structure refinement using the in-plane data alone, which only allows us to determine the projection of the structure, leads to a clear preference for the interstitial model with an $R$ value of 0.12 , for the other models the $R$ values were $R=0.18,0.3$, and 0.5 . It can therefore be clearly concluded that the only model giving convincing agreement between measured and calculated data is the interstitial model.

The best-fit model is shown in Fig. 2. From corresponding $r$ factors summarized in Fig. 3, it is apparent that a fraction of about $50 \%$ brings about the best fit exhibiting $R_{w}$ $=10.6 \%$ and a goodness of fit of 1.7. The value of $50 \%$ interstitials is in good agreement with the DAI model originally proposed for the $\mathrm{Si}(113)-(3 \times 2)$ surface $^{3}$ in Ref. 3 an interstitial atom sits only beneath one of the two pentamers per $(3 \times 2)$ cell. In Table I the best-fit Ge coordinates for the DAI model with 50\% interstitial atoms are compiled; the numbering of the Ge atoms corresponds to that shown in Fig. 2. The theory-experiment agreement can be judged from Fig. 4. Further models tested were an asymmetry model recently proposed from STM measurements, ${ }^{12}$ and buckled dimers in the DA model (i.e., the puckered model ${ }^{12}$ ). The agreement was in both cases significantly worse than with the interstitial model (43\% and 26\%, respectively) and can therefore clearly be ruled out.

From LEED and also from SXRD the $(3 \times 1)$ phase appears very well ordered, i.e., with a low density of antiphase boundaries and steps. This was indicated by the narrow spot profiles reflecting $(3 \times 1)$ domain sizes larger than $2000 \AA$. On the other hand, the $(3 \times 2)$ periodicity is poorly developed, exhibiting diffuse and broad diffraction features. The size of the $(3 \times 2)$ domains derived from the width of the corresponding reflections was about 25-30 $\AA$, consistent with recent spot profile analysis low-energy electrondiffraction (SPALEED) measurements. ${ }^{17}$ This leads to the suggestion that the $(3 \times 2) \rightarrow(3 \times 1)$ transformation is an order-disorder transition belonging to the Ising model; note that intensities of $(3 \times 1)$ reflections are not affected by this disordering process because the interstitials are always in registry with the $(3 \times 1)$ periodicity. Since the SXRD measurements were performed at room temperature, it is hard to differentiate from our measurements whether this disordering is thermally induced by an order-disorder transition or just a frozen-in phase. A recent STM study indicated, however, that at room temperature the $(3 \times 1)$ phase on $\mathrm{Ge}(113)$ 

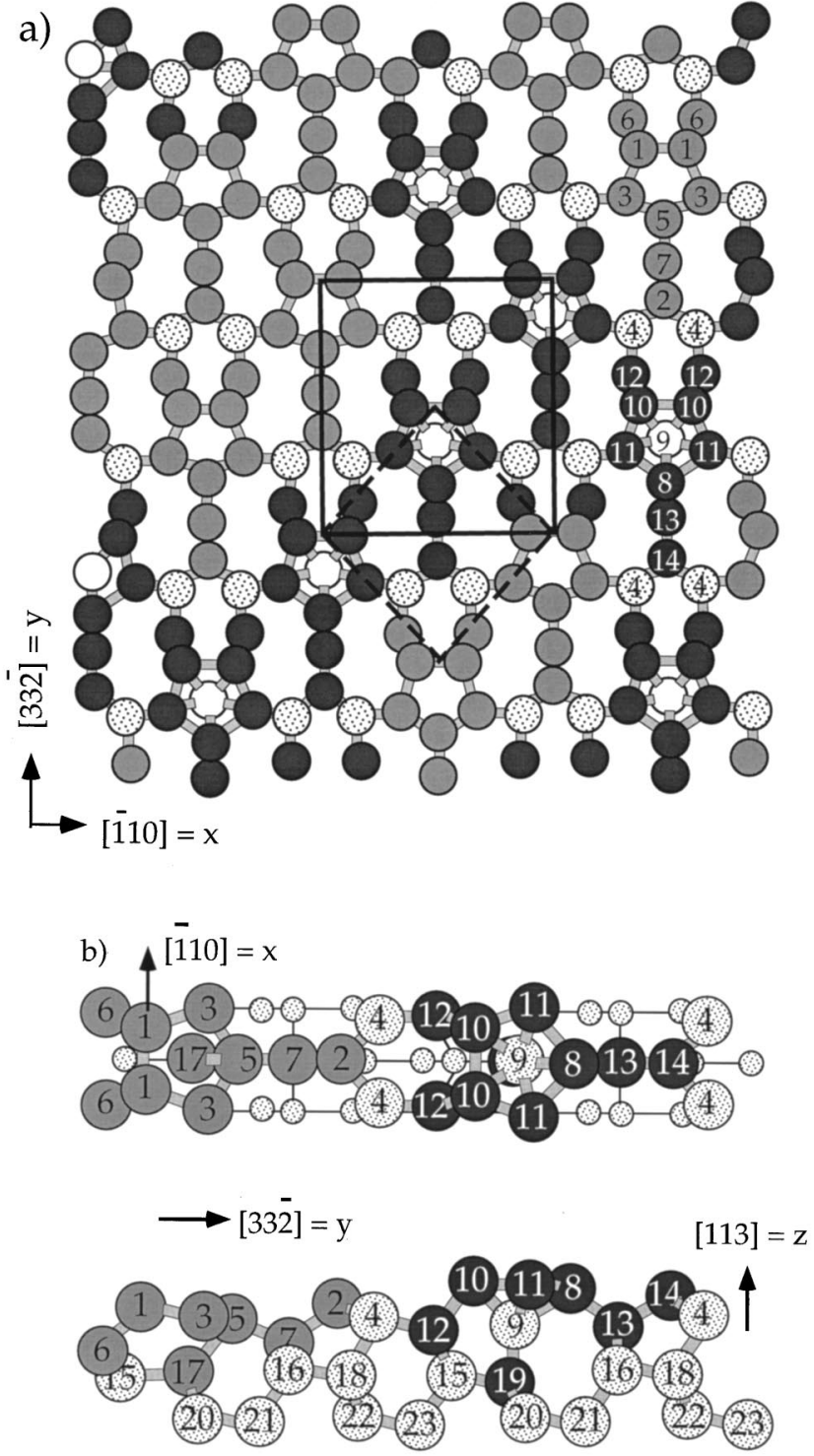

FIG. 2. (a) Top view of the structure of Ge(113) with disordered distribution of interstitials. The $3 \times 2$ unit cell is indicated. (b) Top and side views show a part of the structure containing an unoccupied (left) and one occupied (right) subsurface interstitial site. The numbering of the atoms is used in the text and in Table I. The presence of the interstitial atom 9 causes the pentamer $(8,10,11)$ to move upward by about $1 \AA$ relative to pentamer $(1,3,5)$.

presents a highly disordered $(3 \times 2)$ phase ${ }^{12}$ which is consistent with a frozen-in phase. High-resolution LEED at $80 \mathrm{~K}$ (Ref. 17) demonstrates that the preparation of a betterordered $(3 \times 2)$ structure requires a very slow cooling from $1000 \mathrm{~K}$ to $80 \mathrm{~K}$ over $3 \mathrm{~h}$. This may point toward a low mobility of the interstitial atoms (or to a too-small energy gain by ordering) which prevents the (113) surface from ordering with $(3 \times 2)$ periodicity. Quite surprising is the fact that interstitial atoms are resistant against contamination from the residual gas, since they persist on the surface for several days. Surface contamination may, however, disorder the array of interstitial atoms since $(3 \times 2)$ spots diminished rapidly with time. As pointed out in Ref. 18, the majority of interstitial atoms may stay in the subsurface region rather than dissolve into the bulk, as the formation energy for bulk

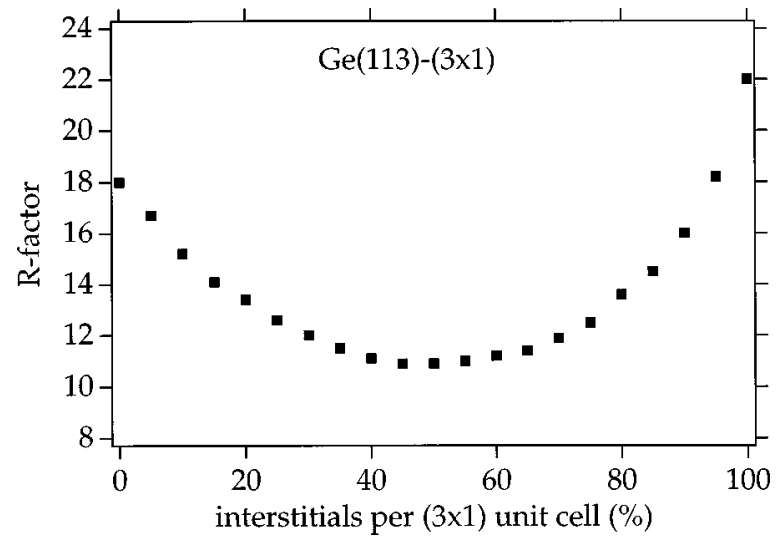

FIG. 3. $R$-factor as a function of the interstitial concentration for the dimer-adatom-interstitial (DAI) model of the Ge(113)- $(3 \times 1)$ reconstruction with a random distribution of interstitials. The optimum concentration is $50 \% \pm 10 \%$.

self-interstitials is too high $(3 \mathrm{eV}$ in $\mathrm{Si}) .{ }^{18}$

Let us now discuss the structural details of the Ge(113)$(3 \times 1)$ surface; in the following the numbers in parentheses are related to Ge atoms as labeled in Fig. 2. The Ge adatom (2) binds to atoms (7) and (4) with bond lengths of 2.30 and $2.49 \AA$, respectively. Both distances are substantially shorter than that observed for $\mathrm{Ge}(111)-c(2 \times 8)\left(2.60 \AA{ }^{19}{ }^{19}\right.$ This might indicate a charge transfer away from the adatom, leaving this atom in a more planar $s p^{2}$-type bond configuration,

TABLE I. Ge coordinates in $\AA$ of the clean reconstructed $\mathrm{Ge}(113)$ surface on the basis of the DAI model with random occupation of subsurface interstitials beneath the Ge tetramers. The numbering of atoms corresponds to that used in Fig. 1. A fixed Debye temperature of $374 \mathrm{~K}$ was used. Numbers with asterisks indicate that those parameters were not optimized.

\begin{tabular}{cccr}
\hline \hline Ge atom & $x$ coordinate & $y$ coordinate & $z$ coordinate \\
\hline 1 & $1.23 \pm 0.04$ & $0.0^{*}$ & $4.35 \pm 0.07$ \\
2 & $6.00^{*}$ & $1.27 \pm 0.07$ & $4.41 \pm 0.11$ \\
3 & $2.02 \pm 0.02$ & $2.52 \pm 0.05$ & $3.95 \pm 0.09$ \\
4 & $4.33 \pm 0.01$ & $2.76 \pm 0.01$ & $3.86 \pm 0.07$ \\
5 & $0.0^{*}$ & $3.66 \pm 0.05$ & $3.80 \pm 0.07$ \\
6 & $4.15 \pm 0.04$ & $5.07 \pm 0.09$ & $2.62 \pm 0.09$ \\
7 & $0.0^{*}$ & $5.92 \pm 0.07$ & $2.91 \pm 0.11$ \\
8 & $0.0^{*}$ & $4.04 \pm 0.05$ & $4.99 \pm 0.09$ \\
9 & $0.0^{*}$ & $1.85 \pm 0.04$ & $3.56 \pm 0.09$ \\
10 & $1.36 \pm 0.04$ & $0.0^{*}$ & $5.29 \pm 0.09$ \\
11 & $2.19 \pm 0.02$ & $2.40 \pm 0.05$ & $5.15 \pm 0.09$ \\
12 & $4.17 \pm 0.04$ & $5.12 \pm 0.10$ & $3.36 \pm 0.09$ \\
13 & $0.0^{*}$ & $5.94 \pm 0.07$ & $3.46 \pm 0.11$ \\
14 & $6.00^{*}$ & $1.38 \pm 0.05$ & $4.73 \pm 0.09$ \\
15 & $6.00^{*}$ & $5.83 \pm 0.05$ & $1.54 \pm 0.09$ \\
16 & $2.01 \pm 0.01$ & $5.93 \pm 0.05$ & $1.77 \pm 0.09$ \\
17 & $0.0^{*}$ & $1.84 \pm 0.01$ & $1.74 \pm 0.15$ \\
18 & $3.90 \pm 0.01$ & $1.73 \pm 0.05$ & $1.57 \pm 0.11$ \\
19 & $0.0^{*}$ & $1.54 \pm 0.12$ & $1.23 \pm 0.11$ \\
20 & $2.0^{*}$ & $2.22 \pm 0.07$ & $-0.16 \pm 0.11$ \\
21 & $2.0^{*}$ & $4.66 \pm 0.05$ & $-0.33 \pm 0.11$ \\
22 & $6.0^{*}$ & $2.12 \pm 0.07$ & $-0.11 \pm 0.13$ \\
23 & $6.00^{*}$ & $4.53 \pm 0.05$ & $-0.49 \pm 0.11$ \\
\hline \hline & & & \\
\hline
\end{tabular}




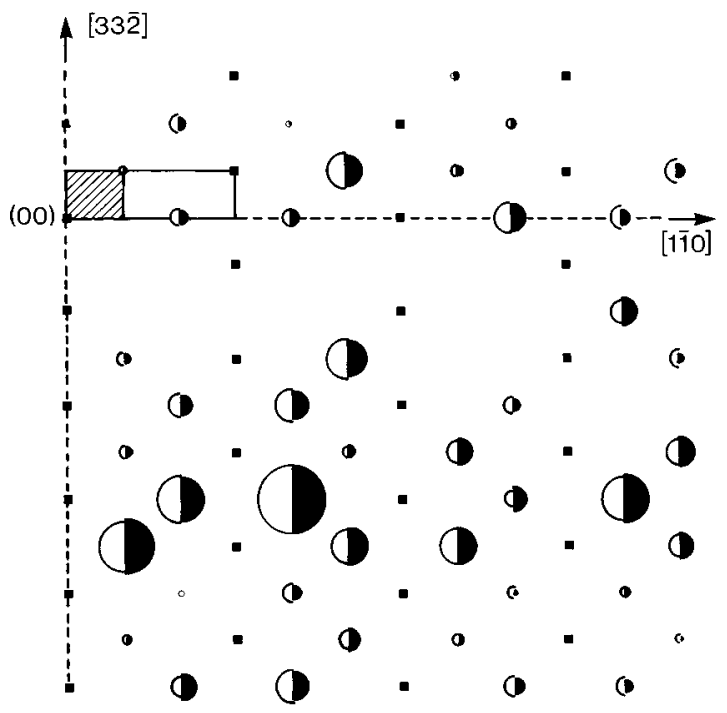

(a)

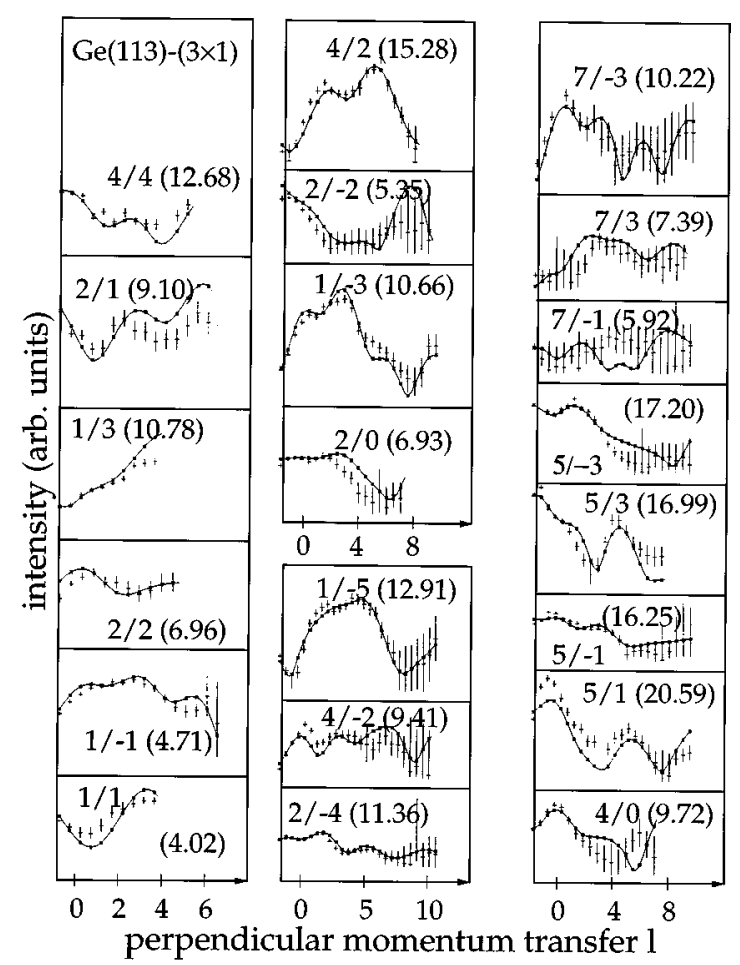

(b)

FIG. 4. (a) Comparison between the measured (filled halfcircles) and calculated (open semicircles) in-plane structure-factor intensities for the DAI model with 50\% interstitals. The radii of the half-circles are proportional to the structure-factor amplitudes, the area to the intensities. (b) Structure-factor intensities of 21 fractional-order rods of the $\mathrm{Ge}(113)-(3 \times 1)$ reconstruction as a function of the perpendicular momentum transfer in units of $b_{3}$. The solid line are calculated intensities for the DAI model with $50 \%$ interstitials and atomic coordinates as given in Table I.

or might indicate contamination by $\mathrm{H}_{2}$ or $\mathrm{H}_{2} \mathrm{O}$. The (001)like Ge atoms (1) and (10) pair up to form dimers, and are part of the five-atom rings, so-called pentamers. While the pentamers without interstitial atoms are buckled by $0.53 \AA$, the pentamers with interstitial atoms are flatter (with a corrugation of only $0.3 \AA$ ). The dimer bond length in the unper- turbed pentamer of $2.47 \AA$ agrees well with the dimer bond lengths found for the $\mathrm{Ge}(001)-(2 \times 1)$ phase $(2.46 \AA) .{ }^{20} \mathrm{By}$ contrast, the dimer bond length in the perturbed pentamer is markedly elongated $(2.72 \AA)$, suggesting that the submerged interstitial atom in this way relieves some of the dimerinduced strain in the surface region. The most prominent distortion due to the insertion of interstitials under the pentamers is the upward shift of the entire pentamer by about 1 $\AA$; this structural feature has not been disclosed by recent DFT calculations. ${ }^{3}$ The relaxation is necessary to accommodate the interstitial atom (9) beneath the pentamer creating bondings to neighboring atoms with reasonable bond lengths. Together with the smaller corrugation of the pentamer [the outward displacement of atom ( 8 ) by $0.2 \AA$ ] the outward shift of the whole pentamer does efficiently relieve the tensile stress introduced by the next adatom in the [33 $\overline{2}]$ direction. The bonding of interstitial (9) to atom (19) is the strongest, with a bond length of $2.35 \pm 0.10 \AA$. Weaker bonds are formed between the interstitial and dimer atoms (2.6-2.8 $\AA$ ).

This current discussion might give the impression that the presence of interstitials on $\mathrm{Ge}(113)$ is strictly related to their high ability to relieve strain in the surface region. If so, the question would arise why the density of interstitials is only $50 \%$ and not $100 \%$. Besides electronic effects, this could be due to repulsion between the interstitial-induced strain fields. However, if mechanical considerations were so important, the interstitials should readily order into a $(3 \times 2)$ network. ${ }^{21}$ Since the $(3 \times 2)$ phase is poorly ordered, electronic effects are expected to play a major role.

From DFT calculations ${ }^{22}$ it follows that $50 \%$ interstitials in the $(3 \times 1)$ cell transforms the otherwise metallic surface into a semiconducting one, which in turn reduces the energy. Disordering of interstitials will leave the Ge(113) surface semiconducting, thus not changing the energetics very much as long as elastic contributions are less important. The pentamer of Ge atoms forms three bonds with the interstitial atom, so that eventually two electrons of this complex are not participating in any bonding. These electrons might be transferred to the adatoms, as suggested in Ref. 22, and consistent with STM images. However, such a charge transfer should move the adatoms outwards rather than inwards as found in the present SXRD analysis. Together with the found interstitial-induced upward shift of the whole pentamer by about $1 \AA$, the present structure analysis raises questions about the electronic structure of that surface, which in turn might trigger further theoretical investigations.

Finally, we should emphasize again that the structural results found for the $\mathrm{Ge}(113)$ surface are very likely to be equally valid for the $\mathrm{Si}(113)$ surface, since electronic properties and the reaction against $\mathrm{H}$ adsorption, as well as their temperature behavior $[(3 \times 1) \rightarrow(1 \times 1)$ phase transition] have been shown to be very much alike. ${ }^{11,23,24}$

In summary, we have shown that the $(3 \times 1)$ reconstruction of $\mathrm{Ge}(113)$ is not only characterized by dimer and adatom motifs reducing the number of dangling bonds, but also exhibits a random distribution of subsurface interstitials. Subsurface interstitials partly relieve the tensile stress introduced by adatoms and dimers, but their main effect is presumably an electronic one in that interstitials make the surface semiconducting regardless of whether they form 
an ordered or a disordered array. Three-dimensional atomic coordinates have been determined by analyzing an extended set of in-plane and out-of-plane x-ray intensity data.

We thank D. Wolf and H. Zajonz for technical support and Karl Jacobi, W. Ranke, and I. K. Robinson for valuable discussions. Special thanks go to J. Dabrowski, who provided the $\mathrm{Si}$ coordinates of $\mathrm{Si}(113)-(3 \times 2)$ found by DFT ground-state calculations (Ref. 3) and many helpful suggestions. Financial support by BMFT (Grant No. 05522WMA) and the Deutsche Forschungsgemeinschaft (SFB 338) is gratefully acknowledged.
${ }^{1}$ B. Z. Olshanetsky and V. I. Mashanov, Surf. Sci. 111, 414 (1981).

${ }^{2}$ J. M. Gibson, M. L. McDonald, and F. C. Unterwald, Phys. Rev. Lett. 55, 1765 (1985); D. J. Eaglesham, A. E. White, L. C. Feldman, N. Moriya, and D. C. Jacobson, ibid. 70, 1643 (1993).

${ }^{3}$ J. Dabrowski, H.-J. Müssig, and G. Wolff, Phys. Rev. Lett. 73, 1660 (1994).

${ }^{4}$ U. Myler and K. Jacobi, Surf. Sci. 220, 353 (1989).

${ }^{5}$ See, e.g., C. B. Duke, Chem. Rev. 96, 1237 (1996).

${ }^{6}$ R. Feidenhans'1, J. S. Pedersen, J. Bohr, M. Nielsen, F. Grey, and R. L. Johnson, Phys. Rev. B 38, 9715 (1988).

${ }^{7}$ K. Takayanagi, Y. Tanishiro, M. Takahashi, and S. Takahashi, J. Vac. Sci. Technol. A 3, 1502 (1985).

${ }^{8}$ R. E. Schlier and H. E. Farnsworth, J. Chem. Phys. 30, 917 (1959).

${ }^{9}$ W. Ranke, Phys. Rev. B 41, 5243 (1990).

${ }^{10}$ H. Huang, S. Y. Tong, U. Myler, and K. Jacobi, Surf. Rev. Lett. 1, 221 (1994).

${ }^{11}$ K. Jacobi and U. Myler, Surf. Sci. 284, 223 (1993).

${ }^{12}$ Zheng Gai, Hang Li, Bo Gao, R. G. Zhao, and W. S. Yang, Phys. Rev. B 54, 8593 (1996).

${ }^{13}$ J. Wang, A. P. Horsfield, D. G. Pettifor, and M. C. Payne, Phys. Rev. B 54, 13744 (1996).

${ }^{14}$ F. Kretschmar, D. Wolf, H. Schulz, H. Huber, and H. Plöckl, Z.
Kristallogr. Mineral. 178, 130 (1987).

${ }^{15}$ R. Feidenhans'1, Surf. Sci. Rep. 10, 105 (1989); I. K. Robinson and D. J. Tweet, Rep. Prog. Phys. 55, 599 (1992).

${ }^{16}$ International Tables for X-Ray Crystallography (Kynoch, Birmingham, 1974), Vol. IV.

${ }^{17}$ A. Iglesias et al. (unpublished).

${ }^{18}$ P. E. Blöchl, E. Smargiassi, R. Car, D. B. Laks, and W. Andreoni, Phys. Rev. Lett. 70, 2435 (1993).

${ }^{19}$ R. G. Silfhout, J. F. Van der Veen, C. Norris, and J. E. Macdonald, Faraday Discuss. Chem. Soc. 89, 169 (1990).

${ }^{20}$ R. Rossmann, H. L. Meyerheim, V. Jahns, J. Wever, W. Moritz, D. Wolf, D. Dornisch, and H. Schulz, Surf. Sci. 279, 199 (1992).

${ }^{21}$ A recent SPALEED investigation by Iglesias et al. (Ref. 17) indicated that, even on very slow cooling from $800 \mathrm{~K}$ down to 80 $\mathrm{K}$, an ordered $3 \times 2$ array is not formed, but rather a striped phase. It consists of $30 \times 100-\AA^{2}$ wide $3 \times 2$ domains which are antiphase arranged along the dimer rows.

${ }^{22}$ J. Dabrowski, H.-J. Müssig, and G. Wolff, J. Vac. Sci. Technol. B 13, 1597 (1995).

${ }^{23}$ W. Ranke and Y. R. Xing, Phys. Rev. B 31, 2246 (1985); S. M. Scholz and K. Jacobi, ibid. 52, 5795 (1995); S. M. Scholz, J. Schreiner, and K. Jacobi, Surf. Sci. 331-333, 402 (1995).

${ }^{24}$ J. Schreiner, K. Jacobi, and W. Selke, Phys. Rev. B 49, 2706 (1994). 